\title{
The intelligent lighting control system of underground garage Guofu Wang ${ }^{a}$,Haidong Liu ${ }^{a}$,Erjie Sun ${ }^{1, a}$ 1630648553@qq.com \\ a JiNan Rail Transit Group Co.,Ltd.
}

Keywords: Underground garage,Intelligent control,ZigBee technology,Path planning.

\begin{abstract}
Traditional underground garage lighting control mainly adopts manual control mode, which usually keep most of the lamps bright for a long time. This way causes a great waste of electricity. To solve the problem, the underground garage lighting intelligent control system is put forward. On the basis of making a rational model for the garage, with the help of a new type of intelligent lighting controller, the classic $\mathrm{A} *$ search algorithm and the existing license plate recognition technology, the system not only can avoid long light phenomenon and control the lighting in the garage reasonably, but also can induce vehicles in the garage to arrive at the designated parking space through the lighting. A specific example of taking advantage of the $\mathrm{A} *$ search algorithm to calculate a path from the entrance to the designated parking space in the underground garage was taken, the simulation results are demonstrated. This shows that the system is feasible and practical.
\end{abstract}

\section{Introduction}

With the development of the society, the number and size of high-rise buildings is increasing, building energy consumption has increased year by year, the situation of building energy saving is grim[1]. In the construction of high-rise building, underground garage is one of the important facilities construction. At present our country building illumination electrical energy consumption accounts for about $10 \%-12 \%$,however the natural lighting of the underground garage is poor, need long time to provide lighting, so lighting energy consumption accounted for the proportion of the energy consumption of the building more enlarged. Underground garage lighting control mode is the traditional manual control, operated by the personnel on duty, a lot of lamps to maintain pilot state in the garage. This approach greatly wasting electric energy and reduce the service of the lamp tube, not only caused a great economic burden to the owners but also do not meet the current requirements of energy saving and environmental protection.

To solve the problems above, this paper will combine the license plate recognition system, the geomagnetic sensor technology, the geomagnetic sensor technology, designed a intelligent lighting control system.

\section{The system composition}

Figure 1 is the underground garage lighting intelligent control system frame. The system is mainly composed of intelligent lighting controller, communication controller, a control center computer. The license plate recognition system is the auxiliary part of this system. 

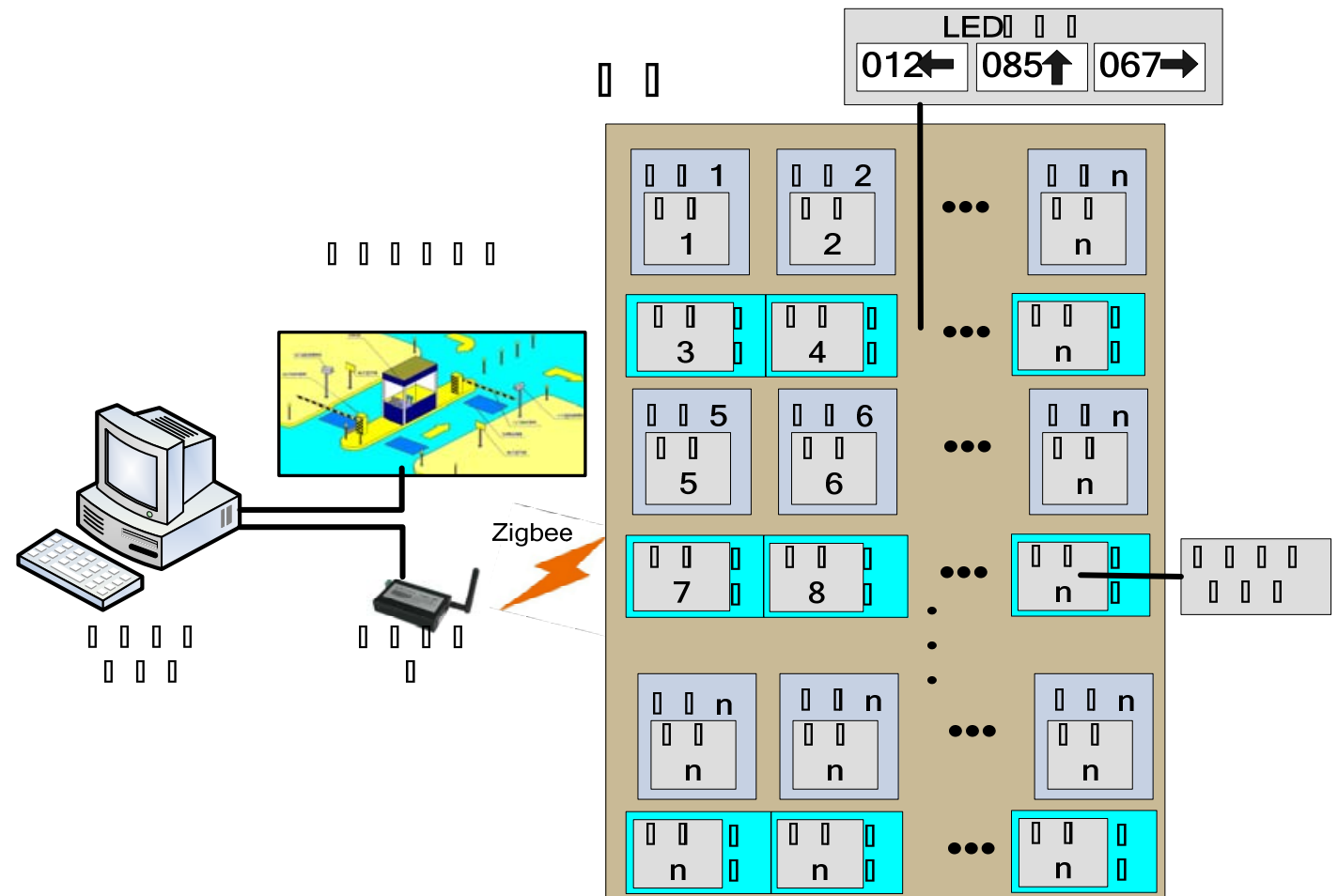

Fig. 1 The structure diagram of underground garage lighting intelligent control system

The main function of the intelligent lighting controller is exchange information with the server and control the navigation light. The hardware of intelligent lighting controller as shown in figure 2 . The ZigBee module is mainly used for communication between intelligent lighting controller and between intelligent lighting controller and communication controller. The sensor is mainly used for the collection of data, which comprises an ultrasonic sensor and a microwave sensor. Ultrasonic sensors are used to detect the direction of the vehicles, the microwave sensor are mainly used to detect if the vehicles are moving. Lighting control module is used to control the lamp light out.

The main function of communication controller is used as a bridge of communication between the control center and intelligent lighting controller. Communication controller is equivalent to a relay station, it between the ZigBee module and the intelligent lighting controller, communication is established, and then through the TCP/IP protocol to establish communication with control center computer.

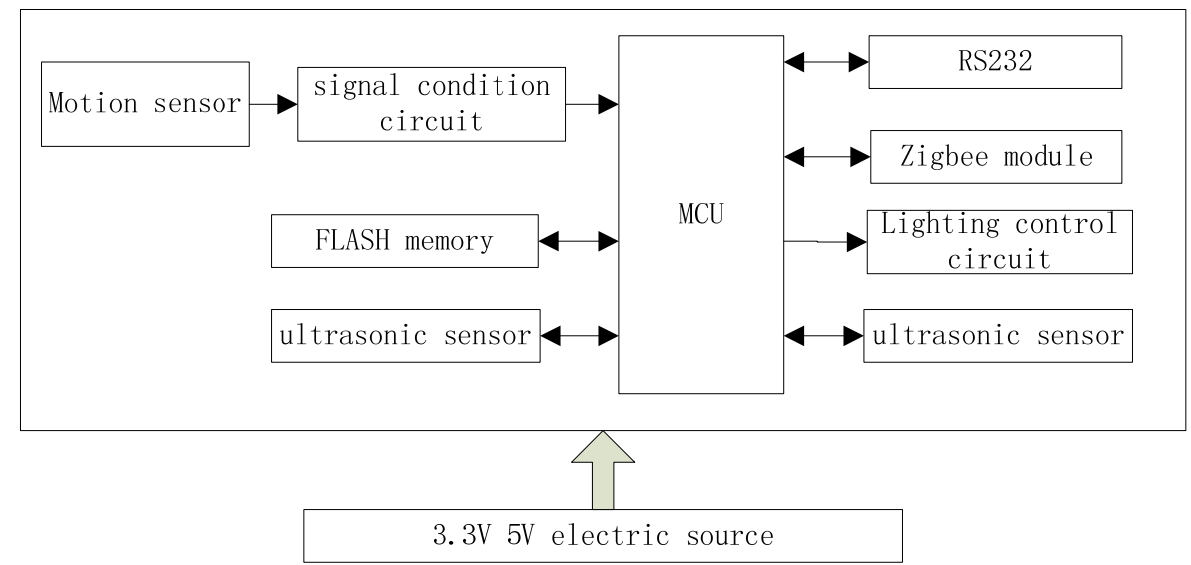

Fig. 2 The hardware constitution of intelligent lighting controller

The main function of control center computer is to store vehicles, parking and other information, license plate recognition, judgment authority for vehicles, vehicle route planning permission, the exchange of information and lighting controller, sent to control the lights out the instructions. 


\section{The communication mode}

The wired communication mode, wiring troubles, and the construction and maintenance cost is high. Wireless sensor network technology is a low-power, self-organizing communication technology, flexible construction, an the cost is low. The system is based on ZigBee technology[2], using the way of combining wired and wireless communication, realized the information interaction between PC and the controller.

The intelligent lighting controllers are arranged in the garage according to certain rules, the mutual communication between ZigBee, so a wireless network is formed. Figure 3 is a block diagram of intelligent lighting controller communication. The intelligent lighting controller collected the information about the vehicle ,then send it to the communication controller through ZigBee, communication controller with Socket communication mode based on TCP/IP protocol to send information to control computer. The computer received the data from communication controller, through analysis these information generate the corresponding control strategy.

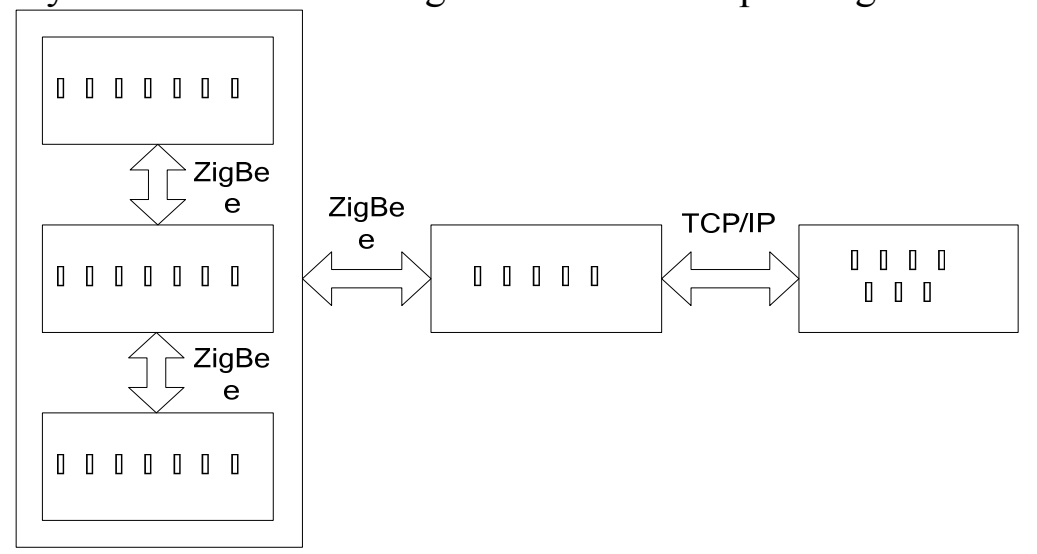

Fig. 3 The communication diagram of intelligent lighting controller

\section{Path planning}

When the vehicles entering or leaving the garage, the computer would plan an optimal path from the starting point to the destination, and at the same time control the corresponding navigation lamps light up.

$4.1 \mathrm{~A} *$ search algorithm

$\mathrm{A}^{*}$ search algorithm is the principle of design of an evaluation function $f(n)=g(n)+h(n), \mathrm{f}(\mathrm{n})$ is the evaluation function of the current node, $g(n)$ represents the actual cost from the initial node to the current node $\mathrm{n}, h(n)$ represents the estimate cost from node $\mathrm{n}$ to the destination node[3]. Through this function can evaluate each point the next step get to, for every search, find the minimum point of the assessed value and continued to search.

The heuristic function $h(n)$ plays a key role on the quality of the choice of path planning[4]. For this study, the sub node has four directions,so using the Manhattan distance as the heuristic function. The expression is $h(n)=\operatorname{abs}\left(n . x-n_{\text {goal }} x\right)+\operatorname{abs}\left(n . y-n_{\text {goal }} y\right), n . x, n . y$ are respectively the $x$ and $y$ coordinates of points $\mathrm{n}, n_{\text {goal }} . x, n_{\text {goal }} y$ are respectively the $x$ and $y$ coordinates of the terminal point.

To calculate the actual cost of $\mathrm{c}\left(\mathrm{n}, \mathrm{n}_{1}\right)$ between two points using Euclidean distance, $c(n, n$ $\left.{ }_{1}\right)=\operatorname{sqrt}\left(\left(n . x-n_{1} \cdot x\right)^{2}+\left(n \cdot y-n_{1} \cdot y\right)^{2}\right), g(n)=c\left(n, n_{1}\right)+g\left(n_{1}\right), \mathrm{n}_{1}$ is the parent node of $\mathrm{b}, g(n 1)$ is the actual distance between starting node and $\mathrm{n}_{1}$.

The specific steps of $\mathrm{A}^{*}$ algorithm are as follows:

(1) Create a table OPEN, a table close,and make two tables empty.And put the starting point $\mathrm{s}$ into the table OPEN.

(2) Search the nodes in the table OPEN, if the table is empty,respecting that the path is not found,failed.

(3) If the table is not empty, then select one from the smallest value of $f$ for the best node, the 
node referred to as a,and put it in CLOSE table.

(4) Judging whether the node a is the destination node e, if the node a is the target node, the seizure of a successful path.

(5) If the node a is not the destination node, then extend it to produce child nodes a1,a2..., perform the following procedure on each child node(in an example of child nodes a1):

(1) If $\mathrm{a}_{1}$ is already in the table OPEN, calculate $\mathrm{g}(\mathrm{a} 1)$, the OPEN table original node al called node ob,compare $g\left(a_{1}\right)$ and $g(o b)$.If $g\left(a_{1}\right)<g(o b)$,modify ob's parent point is a,modified the value of $\mathrm{g}(\mathrm{ob})$,assigned $g\left(a_{1}\right)$ to $\mathrm{g}(\mathrm{ob})$, at the same time update $f(o b)$;if $g\left(a_{1}\right) \geq g(o b)$, then stop expanding node.

(2) If $a_{1}$ is already in the table CLOSE,then skip the node,return(5), continue to expand other nodes.

(3) If $a_{1}$ neither in table OPEN nor table CLOSE,then put it in table OPEN, add a pointer point to its parent node a,calculate $g\left(a_{1}\right)$ 。

(6)Step into step (2) continue to cycle.

\section{Calculation Example}

Under a specific instance in the garage using $A^{*}$ search algorithm to search a path from the entrance to the designated parking spaces 230, and display lighting controller numbers on the path.

According to the garage CAD drawings, can be informed of the actual situation of the environment inside the garage.Because of space, here only display local electrical CAD contains the entrance point and end point of the parking garage, as shown in figure 4.The figure shows the distribution of parking spaces and lamps inside the garage, and according to the requirements allocate the intelligent lighting controllers.In the figure $\longmapsto$ represent lamps, $m$ represent intelligent controllers, the gray boxes represent spaces, the number inside the boxes represent the number of parking spaces.

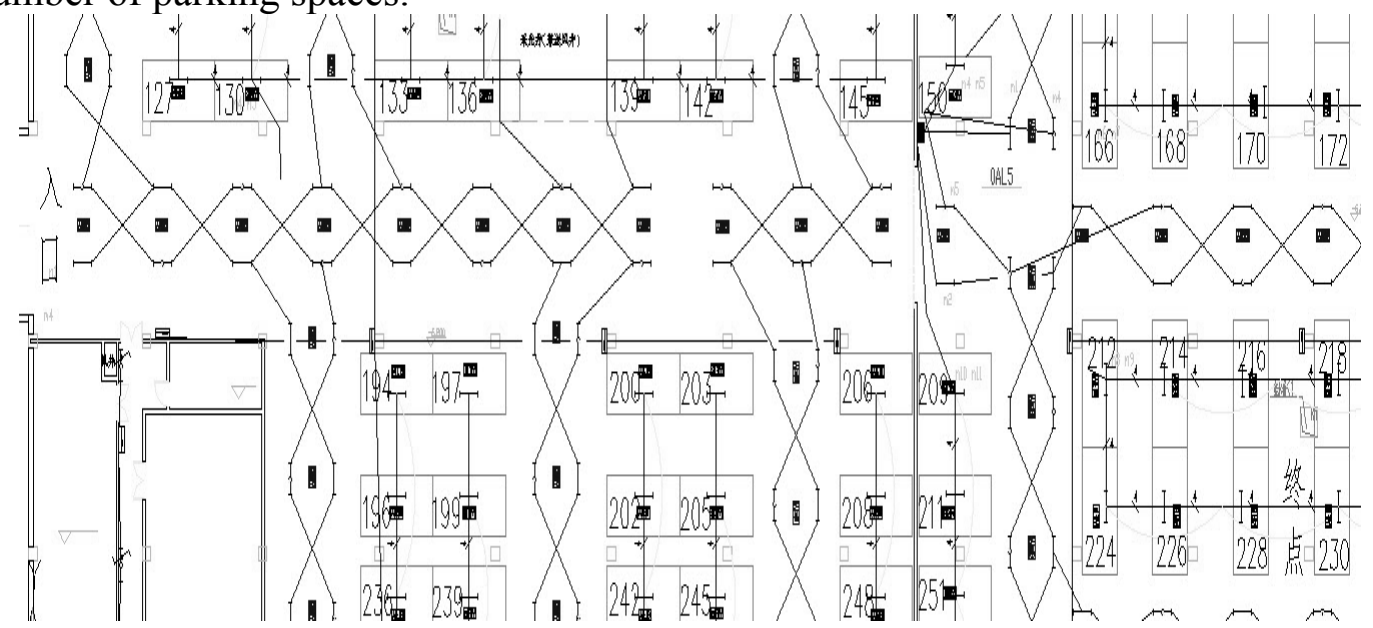

Fig. 4 The electrical CAD diagram of part of a garage

Figure 5 is a garage path planning algorithm simulation map,using Visual C \# compiler tools and object-oriented language $\mathrm{C} \#$,using the grid method for modeling the entire garage.The grid size is $22 * 20$, the dark black grid represents the wall,the dark gray grid represents the intelligent lighting controller on the space.Light gray grid represents the path,white grid represents the intelligent lighting controller on the road, with diagonal grid represents the child nodes not included in the path after the expansion process. 


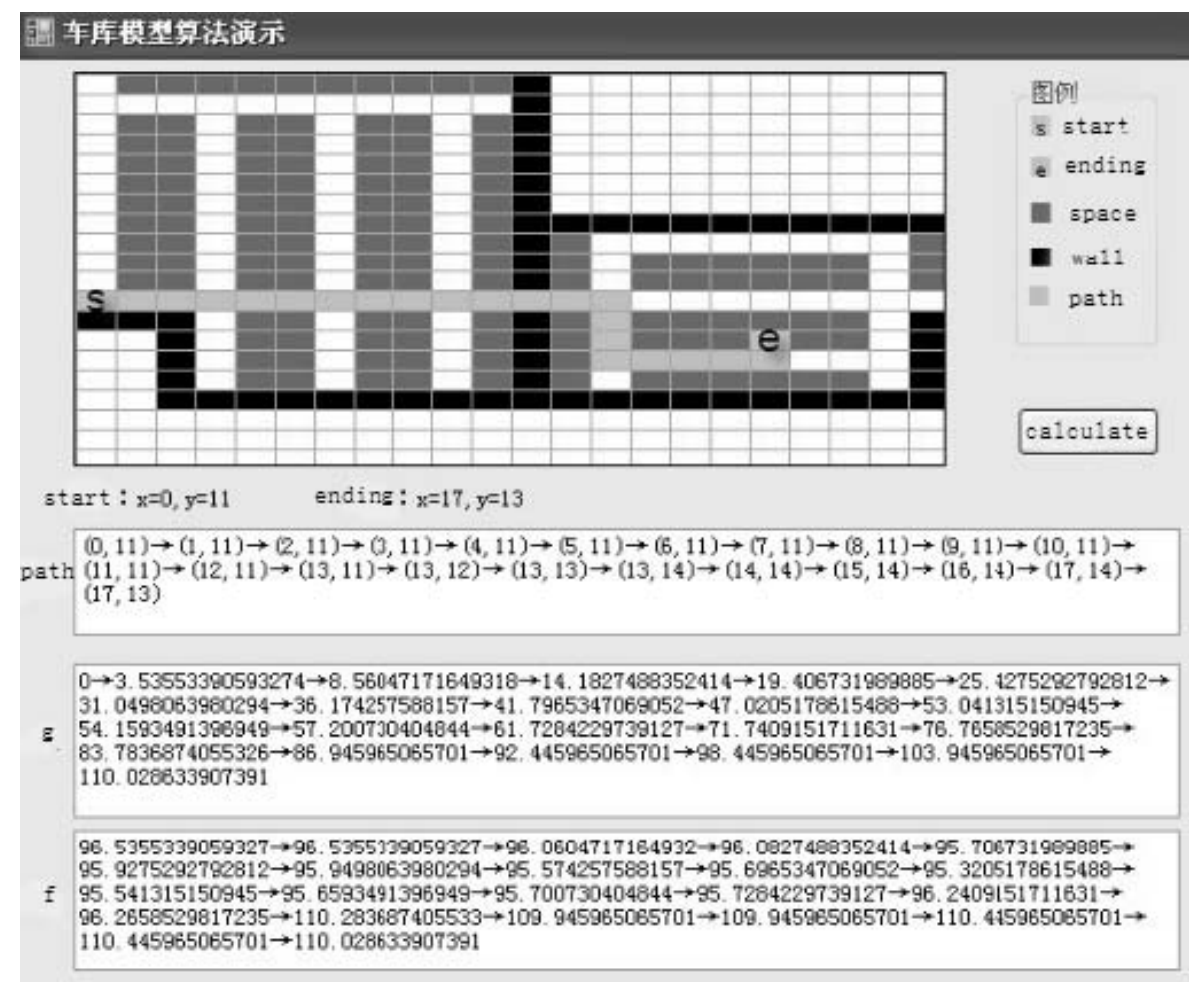

Fig.5 The simulation diagram of path planning algorithm in the garage

In Figure 5 there are four coordinates of each grid, $x, y, x_{1}, y_{1}$ respectively,where $x, y$ are the coordinates of each grid on the simulation map,move one grid to the left, $\mathrm{x}-1$;move one grid to the right, $\mathrm{x}+1$; move down a grid, $\mathrm{y}-1$; move up a grid, $\mathrm{y}+1$. The coordinates of the grid are the marks of each grid,through increase or decrease the coordinates,can present the relationship between each controller and its peripheral controllers.x1, y1 is the actual geographic coordinates which is based on the garage CAD map,in the process of path searching calculate g, $f$ by using $x 1, y 1$.

In order to avoid the car cross over the parking spaces during the process of path planning,so the grids on behalf of the intelligent lighting controllers on the road are always feasible grids, the grids on behalf of the intelligent lighting controllers on the parking spaces are unfeasible grids,after issuing the query command,the target parking space is changed into feasible grid by unfeasible grid.

The path planning calculation are as follows.At first we should determine the coordinates of the starting point and ending point, the corresponding lighting controller of the starting point is $\mathrm{s}(0$, $11)$,its actual coordinates are $(3.7,78.5)$, the corresponding lighting controller of the ending point is $(17,13)$, its actual coordinates are $(86.5,93)$. Put the starting point $s(0,11)$ into the OPEN table,the minimum $\mathrm{f}$ value of the node is $\mathrm{a}=\mathrm{s}$,put $\mathrm{s}$ into CLOSE table, $\mathrm{s}$ is not a target node,

extending it to produce two child nodes, $\mathrm{a}_{1}(0,10)$ and $\mathrm{a}_{2}(0,10)$. Operate on each child node, the node $a_{1}$ neither in table OPEN nor table CLOSE,put it in table OPEN,set $s$ to the parent node of $a_{1}$,call up the actual coordinates of $\mathrm{a}_{1}$, calculate $\mathrm{g}\left(\mathrm{a}_{1}\right), \mathrm{g}\left(\mathrm{a}_{1}\right)=\operatorname{sqrt}\left((3.7-3.7)^{2}+(73-78.5)^{2}\right)=5.5$, calculate

$h(\mathrm{a} 1), \mathrm{h}\left(\mathrm{a}_{1}\right)=\operatorname{abs}(3.7-86.5)+\operatorname{abs}(73-93)=102.8$, calculate $\mathrm{f}\left(\mathrm{a}_{1}\right), \mathrm{f}\left(\mathrm{a}_{1}\right)=5.5+102.8=108.3 ; \quad$ similar operation to $\mathrm{a}_{2}$, its actual coordinates is $(7.5,78.5), \mathrm{g}\left(\mathrm{a}_{2}\right)=\operatorname{sqrt}\left((7.5-3.7)^{2}+(78.5-78.5)^{2}\right)=3.8$, $\mathrm{h}\left(\mathrm{a}_{2}\right)=\operatorname{abs}(7.5-86.5)+\operatorname{abs}(78.5-93)=93.5, \mathrm{f}\left(\mathrm{a}_{2}\right)=3.8+93.5=97.3$. Now, the table OPEN is not empty,he minimum $\mathrm{f}$ value of the node is $\mathrm{a}=\mathrm{a}_{2}$,put $\mathrm{a}_{2}$ into table CLOSE, after judgment that $\mathrm{a}_{2}$ is not the target node,so extend it.Like this calculated it step by step,minimum $f$ value of the node is $a=e(17,13)$ in the table OPEN, it shows that have already found a path successfully.

The specific path as well as the $g$ value, $f$ value of each point on the path are all shown in Figure 5.After verification that, $\mathrm{A}^{*}$ algorithm can find a shortest path quickly and efficiently,once the garage environment changes, can regroup the map quickly, take the current position as a starting point,calling the $\mathrm{A}^{*}$ algorithm again,search the shortest path. 


\section{Summary}

All of the world are advocating energy conservation and environmental protection,building energy conservation can not be ignored.Building energy saving contains a wide range, as general underground garage lighting control mode is backward,power consumption is big,so designed this intelligent lighting control system, it can navigate the vehicle according to control the lights,not only to facilitate the parking, but also to save energy.

\section{References}

[1] Zhu Peng Wang Yanfeng, Huang Jiwen. The illumination energy conservation research of residential underground garage. Electrical Technology of Intelligent building. 2008; 2 (6) : 60-63

[2] Wang Yan, Liu Peng, Jin Shuai. The system design and implementation of remote wireless video monitoring. Scientific Technology and Engineering. 2011; 11 (30) : 7397-7401

[3] Wang Yong. The multiple mobile robot path planning research in intelligent warehouse system. Harbin: Harbin Industrial University. 2010:20-22.

[4]K. R. Harinarayan, V. J. Lumelsky. Sensor-based motion planning for multiple mobile robots in an uncertain environment. IEEE Proceedings of the IEEE/RSJ/GI International Conference on. 2002, 3: 1485-1492. 